\title{
Clinical characteristics and outcomes of adult patients hospitalized with influenza, respiratory syncytial virus and human metapneumovirus infections: a prospective, cohort study
}

Liang Chen ( $\square$ chenliang1995@sina.com )

Beijing Jishuitan Hospital

Xiudi Han

Qingdao Municipal Hospital Group

Lu Bai

Beijing Jishuitan Hospital

Jian Zhang

Beijing Jishuitan Hospital

\section{Research}

Keywords: Influenza, Respiratory syncytial virus, Human metapneumovirus, Adult, Clinical characteritic, Disease severity

Posted Date: October 2nd, 2020

DOI: https://doi.org/10.21203/rs.3.rs-84881/v1

License: (9) This work is licensed under a Creative Commons Attribution 4.0 International License.

Read Full License 


\section{Abstract}

Background Respiratory syncytial virus (RSV), human metapneumovirus (hMPV), and influenza virus infections cause countless adult hospitalizations each year, yet the clinical characteristics and outcomes of RSV and hMPV infections in adults remain poorly understood. This study was thus designed to compare the clinical findings and severity between adult patients hospitalized with RSV/hMPV infections relative to those hospitalized with influenza.

Methods This study prospectively enrolled 594 patients that had been hospitalized with influenza-like illness and laboratory confirmed RSV, hMPV, or influenza viral infections over the course of three consecutive influenza seasons at a tertiary hospital in China. In order to identify clinical features associated with these three viral infections and with disease severity, univariate and multivariate logistic regression analyses were conducted.

Results Myalgia and lymphocyte counts $<0.8 \times 109 / \mathrm{L}$ were positively correlated with the incidence of influenza infection, whereas age $\geq 65$ years, nasal congestion, dyspnea, and the presence of solid malignant tumors were positively associated with RSV or hMPV infections. However, none of these variables exhibited good predictive performance as a means of discriminating among patients infected with these three different viruses (AUC < 0.70 ). After controlling for potential confounding variables, RSV infections in pneumonia patients were associated with a comparable 30-day mortality risk [odds ratio (OR) 1.016, 95\% confidence interval (Cl) 0.267-3.856, $p=0.982$, whereas hMPV infection was associated with a reduced risk of mortality (OR $0.144,95 \% \mathrm{Cl} 0.027-0.780, \mathrm{p}=0.025)$. In patients without pneumonia, however, 30-day mortality risk in patients infected with influenza virus was comparable to that in patients infected with RSV (OR 1.268, 95\% Cl 0.172-9.355, $\mathrm{p}=0.816)$ or hMPV (OR 1.128, 95\% $\mathrm{Cl} 0.122-10.419, \mathrm{p}=$ 0.916).

Conclusions Clinical features of influenza, RSV, and hMPV infections are helpful, but not sufficiently distinct to permit discrimination among these three different infection types, and specific pathogenic testing is thus necessary to understand the etiological basis for disease in patients with influenza-like illness. In addition, disease severity associated with these three types of viral infection was inconsistent when comparing patients with and without pneumonia.

\section{Introduction}

Viral respiratory tract infections (RTIs) are very common, yet result in millions of emergency medical visits and hospitalizations each year, resulting in significant morbidity and mortality globally [1-3]. Respiratory syncytial virus (RSV) and human metapneumovirus (hMPV) are major pathogens responsible for of RTIs in young children [4-5], with most children experiencing a minimum of one RSV or hMPV infection before the age of two [6]. While classically studied in children, recent advances in molecular diagnostic techniques have led clinicians to recognize that RSV and hMPV can infect and cause significant disease in individuals of all ages [7-8]. In the United States, for example, RSV and hMPV were both associated 
with hospitalization rates of 10-20 per 1000 persons over the age of 50 each year [9]. Notably, these rates are very similar to annual influenza-associated hospitalization rates in this same age group [10].

In regions with temperate climates, both RSV and hMPV exhibit defined seasonal patterns that overlap with influenza seasonality, with peak infection rates occurring between late fall and early April [11-12]. RSV and hMPV infections also typically present with symptoms similar to those characteristic of influenza infections, making clinical differentiation between these different infection types challenging. While influenza is typically the subject of significant research and testing, appropriate diagnostic tests for RSV and hMPV are often unavailable in many hospitals, and as such, little data is available regarding adult infections with these two pathogens [13-14].

The present prospective study was conducted in an effort to understand the clinical features and outcomes of patients with community-acquired RSV, hMPV, and influenza infections in order to identify reliable approaches to differentiating between these infections, and to evaluate the relationship between virus type and disease severity.

\section{Patients And Methods}

\section{Study design and population}

Hospitalized patients $\geq 18$ years old with influenza-like illness (ILI) were prospectively enrolled in the present study during three consecutive influenza seasons (from November - March): 2016/2017, 2017/2018, and 2018/2019. This study was conducted at Beijing Jishuitan Hospital, a teaching hospital with 1500 beds, and all patients were evaluated for nine respiratory viruses: influenza virus $A / B$, human rhinovirus/enterovirus, RSV, hMPV, parainfluenza virus 1-4, human adenovirus, human coronavirus (HKU1, NL63, 229E and OC43). All patients found to have laboratory-confirmed cases of RSV, hMPV, or influenza were recruited to the present study. Patients were excluded from this analysis if they had been hospitalized within the last 28 days [15], or if they were coinfected with two or more respiratory viruses. Written informed consent were obtained from parents. Ethical approval was obtained from the Ethics Committee of Beijing Jishuitan Hospital (No. 202006-18).

\section{Sample Collection}

Blood samples, urine samples, and nasopharyngeal (NP) or oropharyngeal (OP) swabs were collected from all patients upon admission, and were transferred to the laboratory on ice for analysis. Sputum was additionally obtained from all patients with a productive cough. Endotracheal aspirates (ETA), bronchoalveolar fluid lavage (BALF), and pleural fluid samples obtained in the course of patient clinical evaluation and care were also assessed as appropriate. Samples were only tested for pathogens if they had been collected within $48 \mathrm{~h}$ of admission.

\section{Pathogen Detection}


Samples were considered positive for a specific virus if viral nucleic acids were detected via real-time polymerase chain reaction (PCR) (The FilmArray Pneumonia panel; BioFire Diagnostics, LLC, Salt Lake City, UT) [16] in analyzed samples.

Patients were considered to suffer from a community-acquired respiratory coinfection if they met the

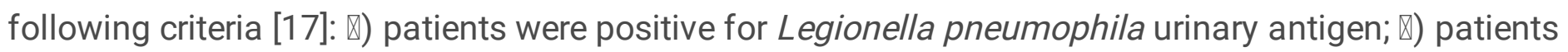
were positive for Streptococcus pneumoniae urinary antigen; 『) patients exhibited positive bacterial cultures from blood or pleural fluid samples, with the exception of coagulase negative Staphylococcus spp.; $\varangle$ ) patients exhibited bacteria in samples of purulent sputum (samples containing $>25$ leukocytes

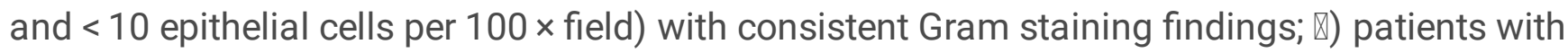
Mycoplasma pneumoniae (MP), Chlamydia pneumonia, or $L$ pneumophila in sputum/BALF/NP/OP swabs as detected via real-time PCR.

\section{Disease And Treatment Definitions}

ILI was defined as a combination of the following [18]: (『) at least one of the following systemic symptoms: fever or feverishness, headache, myalgia or malaise, and (ii) at least one of the following respiratory symptoms: cough, sore throat or dyspnea. Pneumonia was defined based upon radiographic images indicating the presence of newly emergent consolidation or other infiltrates. Systemic corticosteroid use was defined by the use of a minimum of one dose of systemic corticosteroids during hospitalization. Complications associated with influenza, RSV or hMPV infections were defined as a new or exacerbated conditions that were confirmed by radiographic and laboratory testing. Lower respiratory tract (LRT) complications included pneumonia or the worsening of asthma/bronchitis/chronic obstructive pulmonary disease (COPD). Cardiovascular complications included the development or worsening of cardiac events such as acute myocardic infarction or decompensated heart failure.

\section{Data Collection}

A uniform case report form (CRF) was employed to assess the following information from all patients: demographic variables, underlying disease (see Supplementary material 1), clinical symptoms, vital signs, laboratory and radiological test results upon admission, community-acquired respiratory coinfections, management [the administration of neuraminidase inhibitors, antibiotics, systemic corticosteroids], and outcomes [LRT and cardiovascular complications on admission, invasive and noninvasive mechanical ventilation during hospitalization, intensive care unit (ICU) admission, duration of hospitalization and 30-day mortality]. For patients hospitalized for $<30$ days, survival outcomes were assessed via telephone-based follow-up.

\section{Statistical analysis}

Kolmogorov-Smirnov tests were used to assess the normality of all data. Normally and non-normally distributed data are given as means \pm standard deviations and medians, respectively. Continuous variables were analyzed using Student's t-tests and Mann-Whitney U-tests, as appropriate, whereas 
categorical variables were analyzed via Chi-squared tests and Fisher's exact test, respectively. A two-tailed $P<0.05$ was the significance threshold for this study. All statistical analyses were performed using IBM SPSS version 22.0 or MedCalc version 19.0.

Demographic and baseline clinical findings were compared between patients with influenza virus infections and patients with RSV/hMPV infections via a univariate analysis. Any variable that achieved a $P$-value $<0.05$ in this initial analysis was retained for a subsequent multivariate logistic regression analysis that was used to identify predictors of RSV and hMPV infections. The performance of respective predictors was estimated by measuring the area under the receiver-operating characteristic (AUROC) curve, followed by the quantification of sensitivity and specificity measures.

After controlling for confounding variables, a multivariate logistic regression analysis was used to assess the relationship between viral infection type and patient outcomes (invasive ventilation, ICU admission, and 30-day mortality) in patients with and without pneumonia. Potential confounding variables included patient age, sex, obesity, smoking history, influenza vaccination status, systemic corticosteroid or antibiotic use during hospitalization, comorbidities, duration from illness onset to admission, coinfection with other community-acquired pathogens, and use of early neuraminidase inhibitor therapy in patients with influenza [19]. These risk factors have previously been found to be linked to clinical outcomes in patients with influenza and other respiratory viral infections [20-21].

\section{Results}

\section{Study population}

Over the course of the study period, 1491 patients with ILI were identified, of whom 594 were enrolled in the present study. These patients included 421 cases of influenza (303 cases with influenza A, and 118 cases with influenza B), 92 cases of RSV infection, and 81 cases of hMPV infection (Fig. 1).

The median age of this study cohort was 64.0 years old, with $48.3 \%(287 / 594)$ of patients being $\geq$ 65 years old. Of these patients, 53.5\% (318/594) were male. The most prevalent comorbidities in these patients included COPD $(52.7 \%, 313 / 594)$, cardiovascular disease $(44.8 \%, 266 / 594)$, and cerebrovascular disease $(16.3 \%, 97 / 594)$, while just $3.0 \%$ (18/594) of patients were immunocompromised. The most common symptoms at time of admission included cough $(98.1 \%, 583 / 594)$, fever $(71.9 \%, 427 / 594)$, sore throat $(54.2 \%, 322 / 594)$, and dyspnea $(38.6 \%, 229 / 594)$ (Table 1$)$. 
Table 1

Demographic and baseline clinical characteristics of patients with the three viruses infections

\begin{tabular}{|c|c|c|c|c|c|c|}
\hline Variable & $\begin{array}{l}\text { Total } \\
(n=594)\end{array}$ & $\begin{array}{l}\text { Flu } \\
(n=421)\end{array}$ & $\begin{array}{l}\text { RSV } \\
(n=92)\end{array}$ & $\begin{array}{l}P_{1} \\
\text { value }\end{array}$ & $\begin{array}{l}\text { hMPV } \\
(n=81)\end{array}$ & $\begin{array}{l}P_{2} \\
\text { value }\end{array}$ \\
\hline Male $(n, \%)$ & $\begin{array}{l}318 \\
(53.5)\end{array}$ & $\begin{array}{l}217 \\
(51.5)\end{array}$ & $49(53.3)$ & 0.765 & $\begin{array}{l}52 \\
(64.2)\end{array}$ & $0.037^{\#}$ \\
\hline Age (median, IQR, years) & $\begin{array}{l}64.0 \\
(56.8- \\
77.0)\end{array}$ & $\begin{array}{l}60.0 \\
(55.0- \\
67.0)\end{array}$ & $\begin{array}{l}70.0 \\
(64.0- \\
72.0)\end{array}$ & $\begin{array}{l}< \\
0.001\end{array}$ & $\begin{array}{l}69.0 \\
(63.5- \\
77.0)\end{array}$ & $\hat{0} .001$ \\
\hline$\geq 65$ years old $(n, \%)$ & $\begin{array}{l}287 \\
(48.3)\end{array}$ & $\begin{array}{l}163 \\
(38.7)\end{array}$ & $68(73.9)$ & $\begin{array}{l}< \\
0.001^{\#}\end{array}$ & $\begin{array}{l}56 \\
(69.1)\end{array}$ & $<$ \\
\hline $\begin{array}{l}\text { Days from illness onset to } \\
\text { admission (median, IQR) }\end{array}$ & $\begin{array}{l}3.0(2.0- \\
5.0)\end{array}$ & $\begin{array}{l}2.5(1.0- \\
3.0)\end{array}$ & $\begin{array}{l}4.0(3.0- \\
6.0)\end{array}$ & $\hat{0} .001$ & $\begin{array}{l}3.0 \\
(2.0- \\
5.0)\end{array}$ & $\hat{0} .001$ \\
\hline $\begin{array}{l}\text { Influenza vaccination in the } \\
\text { past year }(n, \%)\end{array}$ & $64(10.8)$ & $34(8.1)$ & $16(17.4)$ & 0.006 & $\begin{array}{l}14 \\
(17.3)\end{array}$ & 0.010 \\
\hline \multicolumn{7}{|l|}{ Comorbidities $(n, \%)$} \\
\hline COPD & $\begin{array}{l}313 \\
(52.7)\end{array}$ & $\begin{array}{l}210 \\
(49.9)\end{array}$ & $57(62.0)$ & $0.036^{\#}$ & $\begin{array}{l}46 \\
(56.8)\end{array}$ & 0.255 \\
\hline Cardiovascular disease & $\begin{array}{l}266 \\
(44.8)\end{array}$ & $\begin{array}{l}196 \\
(46.6)\end{array}$ & $39(42.4)$ & 0.468 & $\begin{array}{l}31 \\
(38.3)\end{array}$ & 0.170 \\
\hline Cerebrovascular disease & $97(16.3)$ & $66(15.7)$ & $17(18.5)$ & 0.509 & $\begin{array}{l}14 \\
(17.3)\end{array}$ & 0.717 \\
\hline Diabetes mellitus & $96(16.2)$ & $64(15.2)$ & $22(23.9)$ & $0.043^{\#}$ & $\begin{array}{l}10 \\
(12.3)\end{array}$ & 0.507 \\
\hline Chronic kidney disease & $36(6.1)$ & $20(4.8)$ & $11(12.0)$ & $0.009^{\#}$ & $5(6.2)$ & 0.795 \\
\hline Solid malignant tumor & $38(6.4)$ & $21(5.0)$ & $12(13.0)$ & $0.004^{\#}$ & $5(6.2)$ & 0.867 \\
\hline Asthma & $30(5.1)$ & $22(5.2)$ & $1(1.1)$ & 0.144 & $7(8.6)$ & 0.344 \\
\hline $\begin{array}{l}\text { Immunocompromised status } \\
(n, \%)\end{array}$ & $18(3.0)$ & $11(2.6)$ & $3(3.3)$ & 1.000 & $4(4.9)$ & 0.260 \\
\hline Obesity $(n, \%)$ & $46(7.7)$ & $31(7.4)$ & $4(4.3)$ & 0.299 & $\begin{array}{l}11 \\
(13.6)\end{array}$ & 0.064 \\
\hline
\end{tabular}

Flu: influenza; RSV: respiratory syncytial virus; hMPV: human metapneumovirus; IQR:; COPD: chronic obstructive pulmonary disease; \#. The values were entered into the multivariate logistic regression model; $p_{1}$ : patients with RSV infection versus patients with Flu; $p_{2}$ : patients with hMPV infection versus patients with Flu; The bolded values are $p$-values $<0.05$, which represented significant differences between two groups. 


\begin{tabular}{|c|c|c|c|c|c|c|}
\hline Variable & $\begin{array}{l}\text { Total } \\
(n=594)\end{array}$ & $\begin{array}{l}\text { Flu } \\
(n=421)\end{array}$ & $\begin{array}{l}\text { RSV } \\
(n=92)\end{array}$ & $\begin{array}{l}P_{1} \\
\text { value }\end{array}$ & $\begin{array}{l}\text { hMPV } \\
(n=81)\end{array}$ & $\begin{array}{l}P_{2} \\
\text { value }\end{array}$ \\
\hline Smoking history $(n, \%)$ & $\begin{array}{l}256 \\
(43.1)\end{array}$ & $\begin{array}{l}172 \\
(40.9)\end{array}$ & $42(45.7)$ & 0.398 & $\begin{array}{l}42 \\
(51.9)\end{array}$ & 0.067 \\
\hline \multicolumn{7}{|l|}{$\begin{array}{l}\text { Baseline clinical features ( } n \text {, } \\
\%)\end{array}$} \\
\hline Fever $\geq 38^{\circ} \mathrm{C}$ & $\begin{array}{l}427 \\
(71.9)\end{array}$ & $\begin{array}{l}311 \\
(73.9)\end{array}$ & $62(67.4)$ & 0.206 & $\begin{array}{l}54 \\
(66.7)\end{array}$ & 0.182 \\
\hline Nosal congestion & $\begin{array}{l}133 \\
(22.4)\end{array}$ & $85(20.2)$ & $35(38.0)$ & $\begin{array}{l}<.001^{\#} \\
\end{array}$ & $\begin{array}{l}17 \\
(21.0)\end{array}$ & 0.870 \\
\hline Rhinorrhea & $\begin{array}{l}153 \\
(25.8)\end{array}$ & $\begin{array}{l}113 \\
(26.8)\end{array}$ & $23(25.0)$ & 0.717 & $\begin{array}{l}17 \\
(21.0)\end{array}$ & 0.271 \\
\hline Sore throat & $\begin{array}{l}322 \\
(54.2)\end{array}$ & $\begin{array}{l}236 \\
(56.1)\end{array}$ & $48(52.2)$ & 0.497 & $\begin{array}{l}38 \\
(46.9)\end{array}$ & 0.130 \\
\hline Myalgia & $\begin{array}{l}203 \\
(34.2)\end{array}$ & $\begin{array}{l}176 \\
(41.8)\end{array}$ & $26(28.3)$ & $0.016^{\#}$ & $\begin{array}{l}16 \\
(19.8)\end{array}$ & $<.001^{\#}$ \\
\hline Cough & $\begin{array}{l}583 \\
(98.1)\end{array}$ & $\begin{array}{l}416 \\
(98.8)\end{array}$ & $90(97.8)$ & 0.460 & $\begin{array}{l}77 \\
(95.1)\end{array}$ & 0.061 \\
\hline Sputum production & $\begin{array}{l}169 \\
(28.5)\end{array}$ & $85(20.2)$ & $12(13.0)$ & 0.113 & $\begin{array}{l}26 \\
(32.1)\end{array}$ & 0.575 \\
\hline Chest pain & $\begin{array}{l}147 \\
(24.7)\end{array}$ & $\begin{array}{l}102 \\
(24.2)\end{array}$ & $23(25.0)$ & 0.876 & $\begin{array}{l}22 \\
(27.2)\end{array}$ & 0.859 \\
\hline Dyspnea & $\begin{array}{l}229 \\
(38.6)\end{array}$ & $\begin{array}{l}133 \\
(31.6)\end{array}$ & $62(67.4)$ & <. 001 & $\begin{array}{l}34 \\
(42.0)\end{array}$ & 0.069 \\
\hline Leukocytes $>10 \times 10^{9} / \mathrm{L}$ & $76(12.8)$ & $54(12.8)$ & $11(12.0)$ & 0.820 & $\begin{array}{l}11 \\
(13.6)\end{array}$ & 0.853 \\
\hline Leukocytes $<4 \times 10^{9} / \mathrm{L}$ & $44(7.4)$ & $28(6.7)$ & $7(7.6)$ & 0.741 & $9(11.1)$ & 0.159 \\
\hline Lymphocytes $<0.8 \times 10^{9} / \mathrm{L}$ & $\begin{array}{l}150 \\
(25.3)\end{array}$ & $\begin{array}{l}124 \\
(29.5)\end{array}$ & 15 (16.3) & $0.010^{\#}$ & $\begin{array}{l}11 \\
(13.6)\end{array}$ & $0.003^{\#}$ \\
\hline Coinfection $(n, \%)$ & $\begin{array}{l}162 \\
(27.3)\end{array}$ & $\begin{array}{l}107 \\
(25.4)\end{array}$ & $18(19.6)$ & 0.236 & $\begin{array}{l}22 \\
(27.2)\end{array}$ & 0.742 \\
\hline \multicolumn{7}{|c|}{$\begin{array}{l}\text { Flu: influenza; RSV: respiratory syncytial virus; hMPV: human metapneumovirus; IQR:; COPD: chronic } \\
\text { obstructive pulmonary disease; \#: The values were entered into the multivariate logistic regression } \\
\text { model; } p_{1} \text { : patients with RSV infection versus patients with Flu; } p_{2} \text { : patients with hMPV infection } \\
\text { versus patients with Flu; The bolded values are p-values }<0.05 \text {, which represented significant } \\
\text { differences between two groups. }\end{array}$} \\
\hline
\end{tabular}


Coinfection with other community-acquired respiratory pathogens was detected in $27.3 \%(162 / 594)$ of patients (Table 1). The most common coinfecting pathogen was Klebsiella pneumoniae $(25.3 \%, 41 / 162)$, followed by Streptococcus pneumoniae $(23.5 \%, 38 / 162)$ and Staphylococcus aureus $(17.9 \%, 29 / 162)$ (Supplementary material 2).

A total of $97.8 \%(581 / 594)$ of patients suffered from LRT complications at time of admission, including pneumonia $(47.1 \%, 280 / 594)$ and acute exacerbation of COPD (AECOPD) $(40.4 \%, 240 / 594)$. Of these patients, $13.6 \%(81 / 594)$ had baseline cardiovascular complications. Antibiotics and systemic corticosteroids were administrated to $98.0 \%(98.0 \%, 582 / 594)$ and $48.0 \%(285 / 594)$ of patients, respectively. Overall, $12.0 \%$ (71/594) of patients underwent invasive ventilation, and $14.1 \%(84 / 594)$ of patients were admitted to ICU. The all-cause 30 -day mortality rate in these patients was $8.4 \%(50 / 594)$. The most common cause of death was severe pneumonia $62.0 \%$ (31/50), followed by heart failure $28.0 \%$ $(14 / 50)$, acute renal failure $4.0 \%(2 / 50)$, septic shock $4.0 \%(2 / 50)$, and acute myocardial infarction $1.0 \%$ (1/50) (Table 2). 
Table 2

Clinical management and outcomes of patients with the three viruses infections

\begin{tabular}{|c|c|c|c|c|c|c|}
\hline Variable & $\begin{array}{l}\text { Total } \\
(n=594)\end{array}$ & $\begin{array}{l}\text { Flu } \\
\left(\begin{array}{c}n= \\
421)\end{array}\right.\end{array}$ & $\begin{array}{l}\text { RSV } \\
(n=92)\end{array}$ & $\begin{array}{l}P_{1} \\
\text { value }\end{array}$ & $\begin{array}{l}\text { hMPV } \\
(n=81)\end{array}$ & $\begin{array}{l}P_{2} \\
\text { value }\end{array}$ \\
\hline $\begin{array}{l}\text { Baseline lower respiratory tract } \\
\text { complications }(n, \%)\end{array}$ & $\begin{array}{l}581 \\
(97.8)\end{array}$ & $\begin{array}{l}413 \\
(98.1)\end{array}$ & $89(96.7)$ & 0.675 & $\begin{array}{l}79 \\
(97.5)\end{array}$ & 1.000 \\
\hline Pneumonia & $\begin{array}{l}280 \\
(47.1)\end{array}$ & $\begin{array}{l}184 \\
(43.7)\end{array}$ & $49(53.3)$ & 0.095 & $\begin{array}{l}47 \\
(58.0)\end{array}$ & 0.018 \\
\hline AECOPD & $\begin{array}{l}240 \\
(40.4)\end{array}$ & $\begin{array}{l}187 \\
(44.4)\end{array}$ & $30(32.6)$ & 0.038 & $\begin{array}{l}23 \\
(28.4)\end{array}$ & 0.010 \\
\hline bronchitis & $40(6.7)$ & $23(5.5)$ & $9(9.8)$ & 0.121 & $8(9.9)$ & 0.131 \\
\hline Athma exacerbation & $21(3.5)$ & $19(4.5)$ & $1(1.1)$ & 0.215 & $1(1.2)$ & 0.284 \\
\hline $\begin{array}{l}\text { Baseline cardiovascular } \\
\text { complications }(n, \%)\end{array}$ & $81(13.6)$ & $\begin{array}{l}52 \\
(12.4)\end{array}$ & $15(16.3)$ & 0.306 & $\begin{array}{l}14 \\
(17.3)\end{array}$ & 0.229 \\
\hline Decompensated heart failure & $75(12.6)$ & $\begin{array}{l}49 \\
(11.6)\end{array}$ & $14(15.2)$ & 0.009 & $\begin{array}{l}12 \\
(14.8)\end{array}$ & 0.002 \\
\hline Acute myocardial infarction & $6(1.0)$ & $3(0.7)$ & $1(1.1)$ & 0.548 & $2(2.5)$ & 0.185 \\
\hline Early NAI administration $(n, \%)$ & $\begin{array}{l}148 \\
(24.9)\end{array}$ & $\begin{array}{l}101 \\
(24.0)\end{array}$ & $25(27.2)$ & 0.520 & $\begin{array}{l}22 \\
(27.2)\end{array}$ & 0.544 \\
\hline Antibiotics use $(n, \%)$ & $\begin{array}{l}582 \\
(98.0)\end{array}$ & $\begin{array}{l}411 \\
(97.6)\end{array}$ & $\begin{array}{l}92 \\
(100.0)\end{array}$ & 0.282 & $\begin{array}{l}79 \\
(97.5)\end{array}$ & 1.000 \\
\hline $\begin{array}{l}\text { Systemic corticosteroid use ( } n \text {, } \\
\%)\end{array}$ & $\begin{array}{l}285 \\
(48.0)\end{array}$ & $\begin{array}{l}215 \\
(51.1)\end{array}$ & $29(31.5)$ & 0.001 & $\begin{array}{l}41 \\
(50.6)\end{array}$ & 0.941 \\
\hline Noninvasive ventilation $(n, \%)$ & $76(12.8)$ & $\begin{array}{l}44 \\
(10.5)\end{array}$ & $18(19.6)$ & 0.015 & $\begin{array}{l}14 \\
(17.3)\end{array}$ & 0.078 \\
\hline Invasive ventilation $(n, \%)$ & $71(12.0)$ & $39(9.3)$ & $15(16.3)$ & 0.046 & $\begin{array}{l}17 \\
(21.0)\end{array}$ & 0.002 \\
\hline Admittance to ICU $(n, \%)$ & $84(14.1)$ & $\begin{array}{l}45 \\
(10.7)\end{array}$ & $19(20.7)$ & 0.009 & $\begin{array}{l}20 \\
(24.7)\end{array}$ & 0.001 \\
\hline $\begin{array}{l}\text { Length of stay in hospital } \\
\text { (median, IQR, days) }\end{array}$ & $\begin{array}{l}10.0 \\
(8.0- \\
14.0)\end{array}$ & $\begin{array}{l}9.0 \\
(8.0- \\
13.0)\end{array}$ & $\begin{array}{l}14.0 \\
(10.0- \\
19.0)\end{array}$ & $\dot{0} .001$ & $\begin{array}{l}10.0 \\
(9.0- \\
16.0)\end{array}$ & 0.003 \\
\hline 30-day mortality $(n, \%)$ & $50(8.4)$ & $26(6.2)$ & $10(10.9)$ & 0.110 & $\begin{array}{l}14 \\
(17.3)\end{array}$ & 0.001 \\
\hline
\end{tabular}

AECOPD: acute exacerbation of chronic obstructive pulmonary disease; ICU: intensive care unit; AMI: Acute myocardial infarction. The bolded values are p-values $<0.05$, which represented significant differences between two groups. 


\begin{tabular}{|c|c|c|c|c|c|c|}
\hline Variable & $\begin{array}{l}\text { Total } \\
(n=594)\end{array}$ & $\begin{array}{l}\text { Flu } \\
\left(\begin{array}{c}n= \\
421)\end{array}\right.\end{array}$ & $\begin{array}{l}\text { RSV } \\
(n=92)\end{array}$ & $\begin{array}{l}P_{1} \\
\text { value }\end{array}$ & $\begin{array}{l}\text { hMPV } \\
(n=81)\end{array}$ & $\begin{array}{l}P_{2} \\
\text { value }\end{array}$ \\
\hline \multicolumn{3}{|l|}{ Direct cause of death } & & \multicolumn{2}{|l|}{$\hat{0} .001$} & $\hat{0.001}$ \\
\hline Severe pneumonia & $32(64.0)$ & $\begin{array}{l}21 \\
(80.8)\end{array}$ & \multicolumn{2}{|l|}{$6(60.0)$} & \multicolumn{2}{|l|}{$5(35.7)$} \\
\hline Heart failure & $11(22.0)$ & $2(7.7)$ & \multicolumn{2}{|l|}{$3(30.0)$} & \multicolumn{2}{|l|}{$6(42.9)$} \\
\hline Acute renal failure & $2(4.0)$ & $0(0.0)$ & \multicolumn{2}{|l|}{$0(0.0)$} & \multicolumn{2}{|l|}{$2(14.3)$} \\
\hline Septic shock & $3(6.0)$ & $3(11.5)$ & \multicolumn{2}{|l|}{$0(0.0)$} & \multicolumn{2}{|l|}{$0(0.0)$} \\
\hline AMI & $2(4.0)$ & $0(0.0)$ & \multicolumn{2}{|l|}{$1(10.0)$} & \multicolumn{2}{|l|}{$1(7.1)$} \\
\hline $\begin{array}{l}\text { AECOPD: acute exace } \\
\text { Acute myocardial infa } \\
\text { differences between } t\end{array}$ & $\begin{array}{l}\text { nic obstru } \\
\text { ded value }\end{array}$ & $\begin{array}{l}\text { ve pulm } \\
\text { re p-valu }\end{array}$ & $\begin{array}{l}\text { ry disea } \\
0.05, \mathrm{w}\end{array}$ & $\begin{array}{l}\text { U: inte } \\
\text { repres }\end{array}$ & $\begin{array}{l}\text { ive care ur } \\
\text { ted signifi }\end{array}$ & AMI: \\
\hline
\end{tabular}

\section{Predictors Of Demographic And Clinical Features Of RSV Infection}

Relative to patients infected with influenza, patients infected with RSV tended to be older (median: 70.0 vs. 60.0 years old), with significantly more of these patients being $\geq 65$ years old $(73.9 \%$ vs $38.7 \%)$. In addition, more patients with RSV had COPD ( $62.0 \%$ vs $49.9 \%)$, diabetes mellitus ( $23.9 \%$ vs $15.2 \%)$, chronic kidney disease (12.0\% vs $4.8 \%$ ), and solid malignant tumors ( $13.0 \%$ vs $5.0 \%$ ) relative to patients with influenza. Nasal congestion (38.0\% vs $20.2 \%)$ and dyspnea $(67.4 \%$ vs $31.6 \%)$ were more common in RSV patients, whereas myalgia ( $28.3 \%$ vs $41.8 \%)$ and lymphocytes $<0.8 \times 10^{9} / \mathrm{L}(16.3 \%$ vs $29.5 \%)$ were more common in influenza patients relative to RSV patients (Table 1).

A multivariate logistic regression model revealed that relative to influenza, age $\geq 65$ years old [odds ratio (OR) 3.972, 95\% confidence interval $(C l) 2.330-6.769, p<0.001$; sensitivity $66.30 \%$, specificity $61.28 \%$, AUROC $=0.638$, solid malignant tumors $(O R 2.883,95 \% C / 1.203-6.907, p=0.018$; sensitivity $13.04 \%$, specificity 95.10\%, AUROC = 0.540), nasal congestion $(O R 1.868,95 \% C / 1.064-3.279, p=0.030$; sensitivity $40.22 \%$, specificity $79.81 \%$, AUROC $=0.600)$, and dyspnea $(O R 4.834,95 \% C l 2.671-8.750, p<$ 0.001 ; sensitivity $67.39 \%$, specificity $64.80 \%, A U R O C=0.679)$ were positively associated with RSV infection, while myalgia $(O R 0.494,95 \% \mathrm{Cl} 0.275-0.888, p=0.018$; sensitivity $73.91 \%$, specificity $41.81 \%$, AUROC $=0.579)$, and lymphocytes $<0.8 \times 10^{9} / \mathrm{L}(O R 0.411,95 \% \mathrm{Cl} 0.211-0.800, p=0.009$; sensitivity $83.70 \%$, specificity $29.45 \%, A U R O C=0.566$ ) were negatively associated with RSV infections to RSV infection (Fig. 2).

\section{Predictors Of Demographic And Clinical Features Of HBPV Infection}


Relative to patients with influenza, those infected with hMPV were more often male (64.2\% vs $51.5 \%)$ and were older on average (median: 69.0 yrs vs $60.0 \mathrm{yrs}$ ), with an age $\geq 65$ years old being more common in these hMPV patients (69.1\% vs $38.7 \%)$. In contrast, myalgia (19.8\% vs $41.8 \%$ ) and lymphocytes $<0.8 \times$ $10^{9} / \mathrm{L}(13.6 \%$ vs $29.5 \%)$ were less commonly observed in patients with hMPV infections relative to patients with influenza (Table 1).

Multivariate logistic regression analysis indicated that relative to influenza, age $\geq 65$ years old (OR 4.075, 95\% CI 2.394-6.938, $p<0.001$; sensitivity 69.14\%, specificity 61.28\%, AUROC = 0.652) was positively correlated with hMPV infection, whereas myalgia (OR 0.280, 95\% Cl0.151-0.522, $p<0.001$; sensitivity $80.25 \%$, specificity $41.81 \%$, AUROC $=0.610)$ and lymphocytes $<0.8 \times 10^{9} / \mathrm{L}(O R 0.339,95 \% \mathrm{Cl} 0.166-$ $0.692, p=0.003$; sensitivity $86.42 \%$, specificity $29.45 \%$, AUROC $=0.579$ ) were all negatively correlated with hMPV infections (Fig. 3).

\section{The impact of RSV, hMPV, and influenza on clinical outcomes in patients with pneumonia}

In patients with pneumonia, univariate analyses indicated that relative to influenza, RSV infections were associated with similar risks of invasive ventilation (OR 0.929, 95\% Cl0.379-2.274, $p=0.871)$, ICU admission (OR 1.429, 95\% Cl0.659-3.099, $p=0.366)$, and 30-day mortality (OR 1.367, 95\% Cl0.5423.447, $p=0.508)$. In addition, these analyses revealed that relative to influenza, hMPV infections in pneumonia patients were associated with similar risks of invasive ventilation $(O R 1.506,95 \% \mathrm{Cl} 0.672-$ $3.372, p=0.320)$ and ICU admission (OR 1.887, 95\% Cl0.894-3.982, $p=0.096)$, but were associated with an increased risk of 30-day mortality (OR 2.811, 95\% Cl1.259-6.278, $p=0.012)$ (Table 3). 
Table 3

Impact of specific virus type on the clinical outcomes among patients with pneumonia

\begin{tabular}{|c|c|c|c|c|c|c|}
\hline \multirow[t]{2}{*}{$\begin{array}{l}\text { Clinical } \\
\text { outcome }\end{array}$} & \multirow[t]{2}{*}{$\begin{array}{l}\text { Virus } \\
\text { type }\end{array}$} & \multirow{2}{*}{$\begin{array}{l}\text { Cases } \\
(n, \%)\end{array}$} & \multicolumn{2}{|c|}{$\begin{array}{l}\text { Univariate logistic } \\
\text { analysis }\end{array}$} & \multicolumn{2}{|c|}{$\begin{array}{l}\text { Multivariate logistic } \\
\text { analysis }\end{array}$} \\
\hline & & & OR $(95 \% \mathrm{Cl})$ & $\begin{array}{l}P \\
\text { value }\end{array}$ & *aOR $(95 \% \mathrm{Cl})$ & $\begin{array}{l}P \\
\text { value }\end{array}$ \\
\hline \multirow[t]{3}{*}{$\begin{array}{l}\text { Invasive } \\
\text { ventilation }\end{array}$} & Flu & $\begin{array}{l}28 / 184 \\
(15.2)\end{array}$ & ref & & ref & \\
\hline & RSV & 7/49 (14.3) & $\begin{array}{l}0.929(0.379- \\
2.274)\end{array}$ & 0.871 & $\begin{array}{l}0.783(0.231- \\
2.656)\end{array}$ & 0.695 \\
\hline & hMPV & $\begin{array}{l}10 / 47 \\
(21.3)\end{array}$ & $\begin{array}{l}1.506(0.672- \\
3.372)\end{array}$ & 0.320 & $\begin{array}{l}0.206(0.044- \\
0.959)\end{array}$ & 0.044 \\
\hline \multirow[t]{3}{*}{ ICU admission } & Flu & $\begin{array}{l}31 / 189 \\
(16.4)\end{array}$ & ref & & ref & \\
\hline & RSV & $\begin{array}{l}11 / 49 \\
(22.4)\end{array}$ & $\begin{array}{l}1.429(0.659- \\
3.099)\end{array}$ & 0.366 & $\begin{array}{l}1.368(0.470- \\
3.978)\end{array}$ & 0.565 \\
\hline & hMPV & $\begin{array}{l}13 / 47 \\
(27.7)\end{array}$ & $\begin{array}{l}1.887(0.894- \\
3.982)\end{array}$ & 0.096 & $\begin{array}{l}0.311(0.075- \\
1.298)\end{array}$ & 0.109 \\
\hline \multirow[t]{3}{*}{$\begin{array}{l}\text { 30-day } \\
\text { mortality }\end{array}$} & Flu & $\begin{array}{l}20 / 184 \\
(10.9)\end{array}$ & ref & & ref & \\
\hline & RSV & 7/49 (14.3) & $\begin{array}{l}1.367(0.542- \\
3.447)\end{array}$ & 0.508 & $\begin{array}{l}1.016(0.267- \\
3.856)\end{array}$ & 0.982 \\
\hline & hMPV & $\begin{array}{l}12 / 47 \\
(25.5)\end{array}$ & $\begin{array}{l}2.811(1.259- \\
6.278)\end{array}$ & 0.012 & $\begin{array}{l}0.144(0.027- \\
0.780)\end{array}$ & 0.025 \\
\hline
\end{tabular}

After adjusting for confounding variables, multivariate logistic regression analyses suggested that RSV and influenza infections were associated with similar risks of invasive ventilation $(O R 0.783,95 \% \mathrm{Cl}$ $0.231-2.656, p=0.695)$, ICU admission (OR 1.368, 95\% $C / 0.470-3.978, p=0.565)$, and 30-day mortality (OR 1.016, 95\% Cl0.267-3.856, $p=0.982)$, whereas hMPV infections were associated with a similar risk of ICU admission (OR 0.311, 95\% $\mathrm{Cl} 0.075-1.298, p=0.109)$, but with decreased risk of invasive ventilation $(O R 0.206,95 \% \mathrm{Cl} 0.044-0.959, p=0.044)$ and 30-day mortality $(O R 0.144,95 \% \mathrm{Cl} 0.027-$ $0.780, p=0.025)$ relative to influenza (Table 3 ).

Among patients with pneumonia, 30-day mortality rates were comparable in patients infected with RSV and influenza, and rates in both of these patient groups were significantly higher than those in patients with hMPV infections after adjusting for confounders (Fig. 4). 
The impact of RSV, hMPV, and influenza on clinical outcomes in patients without pneumonia

In patients without pneumonia, univariate analyses revealed that RSV infection was associated with increased risks of invasive ventilation (OR 4.696, 95\% $C / 1.766-12.485, p=0.002)$ and ICU admission (OR $3.641,95 \% \mathrm{Cl} 1.424-9.310, p=0.007)$, but with a similar risk for 30-day mortality $(O R 2.887,95 \% \mathrm{Cl}$ $0.694-12.017, p=0.145$ ) relative to influenza infection. Similarly, hMPV infection was associated with an increased risk of invasive ventilation (OR 5.327, 95\% $C / 1.905-14.894, p<0.001)$ and ICU admission (OR $4.130,95 \% \mathrm{Cl} 1.532-11.128, p=0.005)$, but with a similar risk of 30 day mortality $(O R 2.406,95 \% \mathrm{Cl}$ $0.466-12.435, p=0.295$ ) relative to influenza infection (Table 4).

Table 4

Impact of specific virus type on the clinical outcomes among patients without pneumonia

\begin{tabular}{|c|c|c|c|c|c|c|}
\hline \multirow[t]{2}{*}{$\begin{array}{l}\text { Clinical } \\
\text { outcome }\end{array}$} & \multirow[t]{2}{*}{$\begin{array}{l}\text { Virus } \\
\text { type }\end{array}$} & \multirow{2}{*}{$\begin{array}{l}\text { Cases } \\
(n, \%)\end{array}$} & \multicolumn{2}{|c|}{ Univariate logistic analysis } & \multicolumn{2}{|c|}{$\begin{array}{l}\text { Multivariate logistic } \\
\text { analysis }\end{array}$} \\
\hline & & & OR $(95 \% \mathrm{Cl})$ & $\begin{array}{l}P \\
\text { value }\end{array}$ & *aOR $(95 \% \mathrm{Cl})$ & $\begin{array}{l}P \\
\text { value }\end{array}$ \\
\hline \multirow[t]{3}{*}{$\begin{array}{l}\text { Invasive } \\
\text { ventilation }\end{array}$} & Flu & $\begin{array}{l}11 / 237 \\
(4.6)\end{array}$ & ref & & ref & \\
\hline & RSV & $\begin{array}{l}8 / 43 \\
(18.6)\end{array}$ & $\begin{array}{l}4.696(1.766- \\
12.485)\end{array}$ & 0.002 & $\begin{array}{l}2.904(0.795- \\
10.606)\end{array}$ & 0.107 \\
\hline & hMPV & $\begin{array}{l}7 / 34 \\
(20.6)\end{array}$ & $\begin{array}{l}5.327(1.905- \\
14.894)\end{array}$ & $\begin{array}{l}< \\
0.001\end{array}$ & $\begin{array}{l}2.878(0.798- \\
10.375)\end{array}$ & 0.106 \\
\hline \multirow[t]{3}{*}{ ICU admission } & Flu & $\begin{array}{l}14 / 237 \\
(5.9)\end{array}$ & ref & & ref & \\
\hline & RSV & $\begin{array}{l}8 / 43 \\
(18.6)\end{array}$ & $\begin{array}{l}3.641(1.424- \\
9.310)\end{array}$ & 0.007 & $\begin{array}{l}2.533(0.747- \\
8.595)\end{array}$ & 0.136 \\
\hline & hMPV & $\begin{array}{l}7 / 34 \\
(20.6)\end{array}$ & $\begin{array}{l}4.130(1.532- \\
11.128)\end{array}$ & 0.005 & $\begin{array}{l}2.329(0.680- \\
7.977)\end{array}$ & 0.178 \\
\hline \multirow[t]{3}{*}{$\begin{array}{l}\text { 30-day } \\
\text { mortality }\end{array}$} & Flu & $\begin{array}{l}6 / 237 \\
(2.5)\end{array}$ & ref & & ref & \\
\hline & RSV & $3 / 43(7.0)$ & $\begin{array}{l}2.887(0.694- \\
12.017)\end{array}$ & 0.145 & $\begin{array}{l}1.268(0.172- \\
9.355)\end{array}$ & 0.816 \\
\hline & hMPV & 2/34 (5.9) & $\begin{array}{l}2.406(0.466- \\
12.435)\end{array}$ & 0.295 & $\begin{array}{l}1.128(0.122- \\
10.419)\end{array}$ & 0.916 \\
\hline
\end{tabular}

After adjusting for confounding variables, multivariate logistic regression analyses suggested that RSV and influenza infections were associated with similar risks of invasive ventilation (OR2.904, 95\% $\mathrm{Cl}$ $0.795-10.606, p=0.107$ ), ICU admission (OR 2.533, 95\% Cl 0.747-8.595, $p=0.136$ ), and 30-day mortality 
(OR 1.268, 95\% Cl 0.172-9.355, $p=0.816)$ in patients without pneumonia. Similarly, hMPV and influenza infections were associated with comparable risks for invasive ventilation (OR2.878, 95\% Cl0.798$10.375, p=0.106)$, ICU admission (OR 2.329, 95\% $\mathrm{Cl} 0.680-7.977, p=0.178)$, and 30-day mortality ( $O R$ $1.128,95 \% \mathrm{Cl} 0.122-10.419, p=0.916)$ in patients without pneumonia (Table 4).

Rates of 30-day mortality were similar in all patients without pneumonia infected with influenza, RSV, and hMPV infections after adjusting for confounders (Fig. 5).

\section{The impact of RSV, hMPV, and influenza on clinical outcomes in all patients}

Relative to influenza infection, univariate analyses revealed that RSV infection was associated with an increased risk of invasive ventilation (OR 1.908, 95\% $\mathrm{Cl} 1.002-3.633, p=0.049)$ and ICU admission (OR $2.175,95 \% \mathrm{Cl} 1.203-3.931, p=0.010)$, but with a similar risk of 30 -day mortality $(O R 1.853,95 \% \mathrm{Cl}$ $0.806-3.990, p=0.115)$ in the overall patient population. In contrast, hMPV infection was associated with an increased risk of invasive ventilation (OR 2.602, 95\% C/1.905-14.894, $p=0.003$ ), ICU admission (OR $2.740,95 \% C / 1.515-4.953, p=0.001)$, and 30-day mortality (OR3.175, 95\% Cl1.577-6.389, $p=0.001)$ relative to influenza infection in the overall patient population (Supplementary material 3 ).

Relative to influenza infection, risks of invasive ventilation (OR 1.553,95\% Cl0.702-3.436, $p=0.277$; OR $1.616,95 \% \mathrm{Cl} 0.733-3.564, p=0.234$; respectively), ICU admission (OR 1.951, 95\% Cl 0.949-4.013, $p=$ 0.069; OR 1.983, 95\% Cl0.953-4.129, $p=0.067$; respectively), and 30-day mortality (OR $1.658,95 \% \mathrm{Cl}$ $0.665-4.132, p=0.278 ; O R 2.193,95 \% C / 0.914-5.262, p=0.079$; respectively) associated with RSV and hMPV infections were similar in the overall patient population after adjusting for potential confounding variables (Supplementary material 3).

Rates of 30-day mortality were similar in all patients without pneumonia infected with influenza, RSV, and hMPV infections after adjusting for confounders (Supplementary Fig. 1).

\section{Discussion}

In the present study, we conducted a comprehensive analysis of clinical characteristics and outcomes among adult patients hospitalized with RSV, hMPV, and influenza virus infections. While we found that certain clinical features were of some value as a means of differentiating among these three viral infections, none were sufficiently reliable predictors of infection. Additionally, we found that levels of disease severity associated with these three viruses differed in patients with and without pneumonia, such that influenza and RSV infections were more severe than hMPV infections in patients with pneumonia, whereas severity levels were similar for all three viruses in patients without pneumonia.

Prior analyses have shown that influenza is the most common cause of ILI during influenza season, accounting for $50-70 \%$ of such cases [22-23], whereas RSV and hMPV infections account for $3-6 \%$ of cases in previously healthy individuals [24-25]. In contrast, RSV and hMPV infections can be detected in $16-25 \%$ of hospitalized adults $\geq 65$ years old with chronic medical conditions [26-27]. In our study cohort, the median age was 64.0 years and nearly a half of the patients suffered from cardiopulmonary 
disease, likely explaining why RSV and hMPV infections were detected in $16 \%$ and $14 \%$ of the overall study population, respectively. In addition to these population-specific differences, frequencies of viral detection can vary based on the pathogen testing approach employed. Antibody-based tests often underestimate rates of RSV and hMPV infection owing to their low sensitivity [28-29]. In the present study, we utilized nucleic acid-based diagnostic approaches and detected a relatively high frequency of RSV/hMPV-associated hospitalizations in analyzed adults.

We found that clinical features associated with RSV, hMPV, and influenza virus infections were largely similar to one another, although certain features did differ between these three infection types. Consistent with prior study results, we found that patients with RSV and hMPV infections were more likely to be older and to suffer from underlying systemic diseases such as cancer [30-31]. The presence of myalgia was more often associated with influenza [32-33], whereas nasal congestion and dyspnea were more closely linked to RSV infection [34-35]. Indeed, wheezing and dyspnea are more characteristic of RSV infections in both infants and adults [34, 36-37], likely owing to the fact that RSV exhibits a specific tropism for small airway epithelial cells, thus resulting in lower respiratory tract involvement. The inflammation and edema associated with such involvement can cause consequent airflow obstruction [38]. Nasal or lower airway replication of RSV can also induce Th2-type immune responses that are associated with RSVspecific IgE and leukotriene production, leading to bronchospasm. In addition to these demographic and symptomatic findings, we also determined that a lymphocyte count $<0.8 \times 10^{9} / \mathrm{L}$ was of value as a means of differentiating between RSV/hMPV infections and influenza, which was not reflected in prior reports. Lymphopenia occurs in $50-100 \%$ of patients with severe influenza, and is primarily associated with a reduction in T lymphocyte counts in the peripheral blood. Prior studies have also found that lymphocyte counts decline significantly in critically ill patients suffering from RSV and hMPV infections [39-40]. As such lymphopenia was less commonly associated with RSV/hMPV infections relative to influenza infections, it may be valuable for differential diagnosis. While we and others have identified certain clinical features that differentiate RSV/hMPV and influenza virus infections from one another, it remains challenging to reliably discriminate among patients with these three types of viral infections. None of these symptoms or demographic variables offered sufficient sensitivity or specificity as a means of differentiating among individuals infected with these three viruses. This was consistent with the findings of a prior study conducted by Walshey et al [41]. As such, while we believe that certain clinical indications may be of value for patient differential diagnosis, especially in resouce-limited and primary hospitals. However, they are not sufficient to serve as an alternative to molecular pathogen identification.

In our study, $6.2 \%, 10.9 \%$, and $17.3 \%$ of patients hospitalized with influenza, RSV, and hMPV died within 30 days following admission, respectively. These mortality rates were consistent with those in prior studies of older adults hospitalized with these three viral infections [18, 26-27]. Many studies to date have assessed disease severity associated with these three types of viral infections, but the results of these studies have been inconsistent. These discrepancies may be attributable to the fact that clinical outcomes of various infectious diseases are associated with many factors, including hosts, pathogens, and the surrounding environment [42]. Controlling for these condounders is thus essential in order to 
obtain reliable study results. For example, the studies conducted by Bjarnason A et al. [43] and Zhang et al. [44] directly compared patient outcomes associated with respiratory virus infections without controlling for any confounders. Similarly, Zhou et al. [45] only controlled for a limited number of confounding variables in their multivariate regression model, and they detected no differences in severe outcomes. Study population heterogeneity may also contribute to these inconsistent findings. For example, Lee et al. [34] retrospectively reviewed 607 patients with RSV infections and 547 patients with seasonal influenza infections in three hospitals in Hong Kong, and found that $42.3 \%$ and $36.7 \%$ of these patients, respectively, exhibited signs of pneumonia. The overall survival rates in this study did not differ significantly between patients infected with these two viruses, but the authors did not separate patients according to pneumonia status in their analyses. Herein, we found that disease severity for these three types of viral infections was inconsistent when comparing patients with and without pneumonia. In pneumonia patients, influenza and RSV disease severity was similar and more severe than that associated with hMPV infections. In contrast, disease severity was comparable for all three virus types in patients without pneumonia. Similarly, Howard et al. [46] conducted a population-based surveillance study in which they evaluated outcomes among adults with community-acquired pneumonia and found that RSV was associated with increased disease severity relative to hMPV (OR 1.82, 95\% C/1.32-2.50). This may be linked to differences in the pathogenicity or tissue tropisms of these viruses. Other complications beyond pneumonia can also contribute to patient death. Loubet et al. [18] found that hMPV infection was independently associated with acute cardiac failure. The results were in line with our findings. We found that rates of decompensated heart failure was comparable or elevated in patients suffering from hMPV infections relative to patients infected by RSV or influenza virus. Accordingly, patients with RSV and influenza more often died due to severe pneumonia, whereas patients with hMPV infections were more likely to succumb to cardiovascular complications. As such, the mortality rates associated with these three viruses were similar in the overall study population.

There are multiple limitations to the present study. For one, while this study did have a prospective design, it was still a single-center study with a limited sample size. In addition, admission criteria for the patients in the present study were based upon the subjective judgements of attending physicians, potentially introducing selection bias associated with the enrollment of more severely ill patients. Caution should be taken, therefore, when applying these conclusions to outpatient populations. Furthermore, some studies have suggested that there are differences in clinical features and outcomes associated with particular respiratory virus subtypes [47-48]. As such subtyping was not performed in the present study, future research will be necessary in order to fully explore the relationship between viral subtypes, clinical findings, and disease severity.

\section{Conclusions}

In summary, our data suggest that while some clinical features may aid in the differentiation between patients with RSV/hMPV infections and patients suffering from influenza, the predictive performance of these features is limited. Molecular analyses thus remain essential to accurate patient diagnosis and management. We observed inconsistencies with respect to the relative severity of RSV, hMPV, and 
influenza infections when comparing patients with and without pneumonia, but observed similar mortality rates associated with these three viruses in our overall patient population. These findings thus underscore the growing unmet medical and public health need to develop vaccines and therapeutics directed against RSV and hMPV.

\section{Abbreviations}

Flu: influenza; RSV: respiratory syncitial virus; hMPV: human metapneumovirus; ILI: influenza-like illness;Flu-p: influenza-related pneumonia ; RSV-p: respiratory syncitial virus-re;ated pneumonia; hMPV-p: human metapneumovirus-related pneumonia; OR: odd ratio; $95 \% \mathrm{Cl}$ : $95 \%$ confidence interval; AUROC: under the receiver-operating characteristic curve; ICU: intensive care unit; COPD: chronic obstructive pulmonary disease.

\section{Declarations}

\section{Ethics approval and consent to participate}

The study was approved by the Ethics Committee of Beijing Jishuitan Hospital (No. 202006-18). Informed consent was obtained from all patients or guardians of patients for being included in the study.

\section{Consent for publication}

Not applicable

\section{Availability of data and materials}

The datasets used and/or analysed during the current study are available from the corresponding author on reasonable request.

\section{Competing interests}

The authors declare that they have no competing interests.

\section{Funding}

This study was founded by Beijing JST research (ZR-201921). The sponsor had no role in study design; the collection, analysis and interpretation of data; the writing of the report; and the decision to submit the article for publication.

\section{Authors' contributions}

Study concept and design: LC, XdH. Acquisition of data: LC, XdH, LB and JZ. Statistical analysis of data: LC. Drafting of the manuscript: LC. Critical revision of the manuscript for important intellectual content: $\mathrm{XdH}$. All authors agree with the article submission. All authors read and approved the final manuscript. 
Acknowledgements

The authors would like to express their gratitude to BMCSCI (http://www.bmcscience.com/) for the expert linguistic services provided.

\section{Author details}

1. Department of Infectious Disease, Beijing Jishuitan Hospital, 4th Medical College of Peking University, Beijing, China. ${ }^{2}$. Department of Pulmonary and Critical Care Medicine, Qingdao Municipal Hospital, Qingdao City, Shandong Province, China.

\section{References}

1. Angela R. Branche, Ann R. Falsey. Parainfluenza Virus Infection.Semin Respir Crit Care Med 2016;37(4):538-54.

2. Jin S, Li J, Cai R, Wang X, Gu Z, Yu H, et al. Age- and sex-specific excess mortality associated with influenza in Shanghai, China, 2010-2015. Int J Infect Dis 2020;98:382-9.

3. Nam HH, Ison MG. Respiratory syncytial virus infection in adults. BMJ 2019;366:15021.

4. Fergie J, Suh M, Jiang X, Fryzek JP, Gonzales T. Respiratory Syncytial Virus and All-Cause Bronchiolitis Hospitalizations Among Preterm Infants Using the Pediatric Health Information System (PHIS). J Infect Dis 2020;jiaa435.

5. Barrera-Badillo G, Olivares-Flores B, Ruiz-López A, Fierro-Valdez MÁ, Gutiérrez-Vargas RI, LópezMartínez I. Human Metapneumovirus: Etiological Agent of Severe Acute Respiratory Infections in Hospitalized and Deceased Patients with a Negative Diagnosis of Influenza. Pathogens 2020;9(2).pii: E85.

6. Moe N, Stenseng IH, Krokstad S, Christensen A, Skanke LH, Risnes KR, et al. The Burden of Human Metapneumovirus and Respiratory Syncytial Virus Infections in Hospitalized Norwegian Children. J Infect Dis 2017;216(1):110-6.

7. Falsey AR, McElhaney JE, Beran J, van Essen GA, Duval X, Esen M, et al. Respiratory syncytial virus and other respiratory viral infections in older adults with moderate to severe influenza-like illness. $J$ Infect Dis 2014;209(12):1873-81.

8. Widmer K, Zhu Y, Williams JV, Griffin MR, Edwards KM, Talbot H.. Rates of hospitalizations for respiratory syncytial virus, human metapneumovirus, and influenza virus in older adults. $J$ Infect Dis 2012;206(1):56-62.

9. Widmer K, Griffin MR, Zhu Y, Williams JV, Talbot HK. Respiratory syncytial virus- and human metapneumovirus-associated emergency department and hospital burden in adults. Influenza Other Respir Viruses 2014;8(3):347-52.

10. Jules A, Grijalva CG, Zhu Y, Talbot KH, Williams JV, Dupont WD, et al. Estimating age-specific influenza-related hospitalization rates during the pandemic (H1N1) 2009 in Davidson Co, TN. 
Influenza Other Respir Viruses 2012;6(3):e63-71.

11. Yu J, Liu C, Xiao Y, Xiang Z, Zhou H, Chen L, et al. Respiratory Syncytial Virus Seasonality, Beijing, China, 2007-2015. Emerg Infect Dis 2019;25(6):1127-35.

12. Price RHM, Graham C, Ramalingam S. Association between viral seasonality and meteorological factors. Sci Rep 2019;9(1):929.

13. Tang JW, Lam TT, Zaraket H, Lipkin WI, Drews SJ, Hatchette TF, et al. Global epidemiology of noninfluenza RNA respiratory viruses: data gaps and a growing need for surveillance. Lancet Infect Dis 2017;17(10):e320-6.

14. Kim L, Rha B, Abramson JS, Anderson LJ, Byington CL, Chen GL, et al. Identifying Gaps in Respiratory Syncytial Virus Disease Epidemiology in the United States Prior to the Introduction of Vaccines. Clin Infect Dis 2017;65(6):1020-5.

15. Chen L, Zhou F, Li H, Xing X, Han X, Wang Y, et al. Disease characteristics and management of hospitalised adolescents and adults with community-acquired pneumonia in China: a retrospective multicentre survey. BMJ Open 2018;8: e018709.

16. Murphy CN, Fowler R, Balada-Llasat JM, Carroll A, Stone H, Akerele O, et al. Multicenter Evaluation of the BioFire FilmArray Pneumonia/Pneumonia Plus Panel for Detection and Quantification of Agents of Lower Respiratory Tract Infection. J Clin Microbiol 2020;58(7):e00128-20.

17. Matias G, Taylor R, Haguinet F, Schuck-Paim C, Lustig R, Shinde V. Estimates of hospitalization attributable to influenza and RSV in the US during 1997-2009, by age and risk status. BMC Public Health 2017;17(1):271.

18. Loubet P, Mathieu P, Lenzi N, Galtier F, Lainé F, Lesieur Z, et al. Characteristics of human metapneumovirus infection in adults hospitalized for community-acquired influenza-like illness in France, 2012-2018: a retrospective observational study. Clin Microbiol Infect 2020;S1198743X(20)30190-7.

19. Brendish NJ, Clark TW. Antiviral treatment of severe non-influenza respiratory virus infection. Curr Opin Infect Dis 2017;30:573-8.

20. Muthuri SG, Venkatesan S, Myles PR, Leonardi-Bee J, Lim WS, Al Mamun A, et al. Impact of

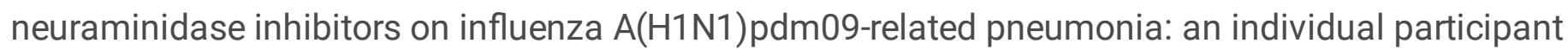
data meta-analysis. Influenza Other Respir Viruses 2016;10:192-204.

21. Guo L, Wei D, Zhang X, Wu Y, Li Q, Zhou M, et al. Clinical Features Predicting Mortality Risk in Patients With Viral Pneumonia: The MuLBSTA Score. Front Microbiol 2019;10:2752.

22. Chittaganpitch M, Waicharoen S, Yingyong T, Praphasiri P, Sangkitporn S, Olsen SJ, et al. Viral etiologies of influenza-like illness and severe acute respiratory infections in Thailand. Influenza Other Respir Viruses 2018;12(4):482-9.

23. Nguyen YT, Graitcer SB, Nguyen TH, Tran DN, Pham TD, Le MT, et al. National surveillance for influenza and influenza-like illness in Vietnam, 2006-2010. Vaccine 2013;31(40):4368-74.

24. Falsey AR, Hennessey PA, Formica MA, Cox C, Walsh EE. Respiratory syncytial virus infection in elderly and high-risk adults. N Engl J Med 2005;352(17):1749-59. 
25. Lefebvre A, Manoha C, Bour JB, Abbas R, Fournel I, Tiv M, et al. Human metapneumovirus in patients hospitalized with acute respiratory infections: A meta-analysis. J Clin Virol 2016;81:68-77.

26. Souty C, Masse S, Valette M, Behillil S, Bonmarin I, Pino C, et al. Baseline characteristics and clinical symptoms related to respiratory viruses identified among patients presenting with influenza-like illness in primary care. Clin Microbiol Infect 2019;25(9):1147-53.

27. Ackerson B, Tseng HF, Sy LS, Solano Z, Slezak J, Luo Y, et al. Severe Morbidity and Mortality Associated With Respiratory Syncytial Virus Versus Influenza Infection in Hospitalized Older Adults. Clin Infect Dis 2019;69(2):197-203.

28. Casiano-Colón AE, Hulbert BB, Mayer TK, Walsh EE, Falsey AR. Lack of sensitivity of rapid antigen tests for the diagnosis of respiratory syncytial virus infection in adults. J Clin Virol 2003;28(2):16974.

29. Falsey AR, Formica MA, Walsh EE. Diagnosis of respiratory syncytial virus infection: comparison of reverse transcription-PCR to viral culture and serology in adults with respiratory illness. J Clin Microbiol 2002;40(3):817-20.

30. Kim YJ, Lee ES, Lee YS. High mortality from viral pneumonia in patients with cancer. Infect Dis (Lond) 2019;51(7):502-9.

31. Yoon JG, Noh JY, Choi WS, Park JJ, Suh YB, Song JY, et al. Clinical characteristics and disease burden of respiratory syncytial virus infection among hospitalized adults. Sci Rep. 2020;10(1):12106.

32. Jennings LC, Anderson TP, Beynon KA, Chua A, Laing RT, Werno AM, et al. Incidence and characteristics of viral community acquired pneumonia in adults. Thorax 2008;63(1):42-8.

33. Sundaram ME, Meece JK, Sifakis F, Gasser RA Jr, Belongia EA. Medically attended respiratory syncytial virus infections in adults aged $\geq 50$ years: clinical characteristics and outcomes. Clin Infect Dis 2014;58(3):342-9.

34. Lee N, Lui GC, Wong KT, Li TC, Tse EC, Chan JY, et al. High morbidity and mortality in adults hospitalized for respiratory syncytial virus infections. Clin Infect Dis 2013;57(8):1069-77.

35. Esteban I, Stein RT, Polack FP. A Durable Relationship: Respiratory Syncytial Virus Bronchiolitis and Asthma Past Their Golden Anniversary. Vaccines (Basel) 2020; 8:E201.

36. Kieke BA, Belongia EA, McClure DL, Shinde V. Prediction of serious RSV-related outcomes in older adults with outpatient RSV respiratory illness during 12 consecutive seasons. Influenza Other Respir Viruses 2020;14(5):479-82.

37. Garofalo RP, Patti J, Hintz KA, Hill V, Ogra PL, Welliver RC. Macrophage Inflammatory protein-1alpha (Not T Helper Type 2 Cytokines) Is Associated With Severe Forms of Respiratory Syncytial Virus Bronchiolitis. J Infect Dis 2001;184:393-9.

38. O'Donnell DR, Carrington D. Peripheral blood lymphopenia and neutrophilia in children with severe respiratory syncytial virus disease. Pediatr Pulmonol 2002;34(2):128-30.

39. Poutanen SM, Low DE, Henry B, Finkelstein S, Rose D, Green K, et al. Identification of severe acute respiratory syndrome in Canada. N Engl J Med 2003;348(20):1995-2005. 
40. Foulongne V, Lechiche C, Reynes J, Segondy M. Human metapneumovirus pneumonia in an adult patient hospitalized for suspected severe acute respiratory syndrome (SARS). Presse Med 2004;33(15):1006-7.

41. Walsh EE, Peterson DR, Falsey AR. Is clinical recognition of respiratory syncytial virus infection in hospitalized elderly and high-risk adults possible? J Infect Dis 2007;195(7):1046-51.

42. Roberts AJ1, Wiedmann M. Pathogen, host and environmental factors contributing to the pathogenesis of listeriosis. Cell Mol Life Sci 2003;60(5):904-18.

43. Bjarnason A, Westin J, Lindh M, Andersson LM, Kristinsson KG, Löve A, et al. Incidence, Etiology, and Outcomes of Community-Acquired Pneumonia: A Population-Based Study. Open Forum Infect Dis. 2018;5:ofy010.

44. Zhang Y, Wang Y, Zhao J, Xiong Z, Fan Y, Zhang W, et al. Severity and mortality of respiratory syncytial virus vs influenza A infection in hospitalized adults in China. Influenza Other Respir Viruses 2020;14(5):483-90

45. Zhou F, Wang Y, Liu Y, Liu X, Gu L, Zhang X, et al. Disease severity and clinical outcomes of community-acquired pneumonia caused by non-influenza respiratory viruses in adults: a multicentre prospective registry study from the CAP-China Network. Eur Respir J 2019;54(2).pii:1802406.

46. Howard LM, Edwards KM, Zhu Y, Grijalva CG, Self WH, Jain S, et al. Clinical Features of Human Metapneumovirus-Associated Community-Acquired Pneumonia Hospitalizations. Clin Infect Dis 2020;ciaa088.

47. Minney-Smith CA, Selvey LA, Levy A, Smith DW, et al. Post-pandemic influenza A/H1N1pdm09 is associated with more severe outcomes than $\mathrm{A} / \mathrm{H} 3 \mathrm{~N} 2$ and other respiratory viruses in adult hospitalisations. Epidemiol Infect 2019; 147:e310.

48. Borchers AT, Chang C, Gershwin ME, Gershwin LJ. Respiratory syncytial virus--a comprehensive review. Clin Rev Allergy Immunol 2013;45(3):331-7.

\section{Figures}




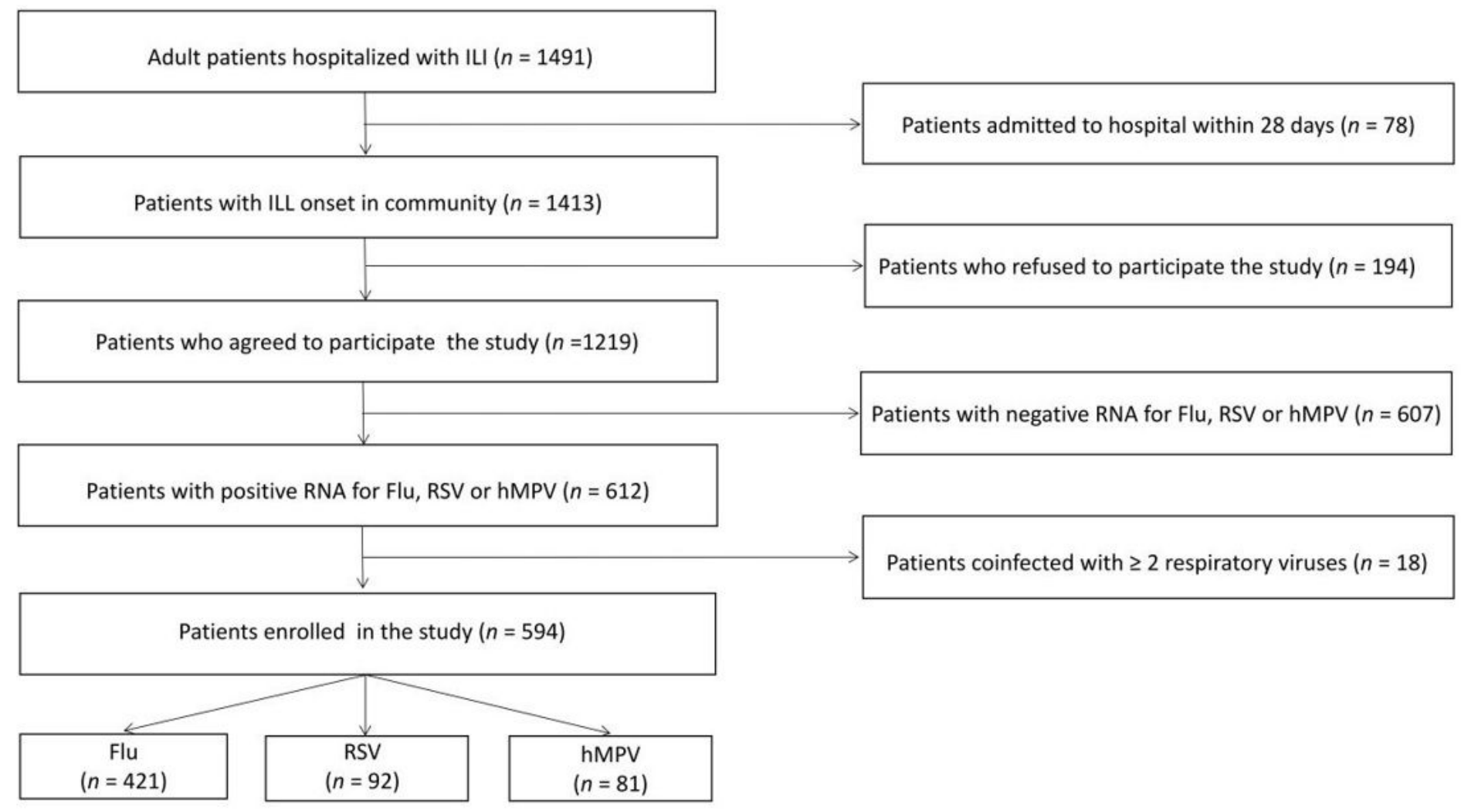

\section{Figure 1}

Flow chart of participant enrollment.1491 patients with ILI were identified, of whom 594 were enrolled in the present study.

\begin{tabular}{|c|c|c|c|c|c|c|}
\hline & & OR $(95 \% \mathrm{Cl})$ & $p$ value & Sensitivity (\%) & Specificity (\%) & AUC \\
\hline Age $\geqslant 65$ years old & $\longrightarrow$ & $3.972(2.330-6.769)$ & $<0.001$ & 66.30 & 61.28 & 0.638 \\
\hline Solid malignant tumor & & $2.883(1.203-6.907)$ & 0.018 & 13.04 & 95.10 & 0.540 \\
\hline Nosal congestion & $\longrightarrow$ & $1.868(1.064-3.279)$ & 0.030 & 40.22 & 79.81 & 0.600 \\
\hline Myalgia & & $0.494(0.275-0.888)$ & 0.018 & 79.31 & 41.81 & 0.579 \\
\hline Dyspnea & & $4.834(2.671-8.750)$ & $<0.001$ & 67.39 & 64.8 & 0.679 \\
\hline Lymphocytes $<0.8 \times 10^{9} / \mathrm{L}$ & & $0.411(0.211-0.800)$ & 0.009 & 83.70 & 29.45 & 0.566 \\
\hline 0.1 & 1 & & & & & \\
\hline Favor Flu & & Favor RSV & & & & \\
\hline
\end{tabular}

\section{Figure 2}


Predictors for Flu and RSV infection. Age $\geq 65$ years old, solid malignant tumors, nasal congestion, and dyspnea were positively associated with RSV infection, while myalgia and lymphocytes < $0.8 \times 109 /$ LNwere negatively associated with RSV infections to RSV infection. None of these variables exhibited good predictive performance as a means of discriminating (area under the receiver-operating characteristic curve $<0.70$ ).

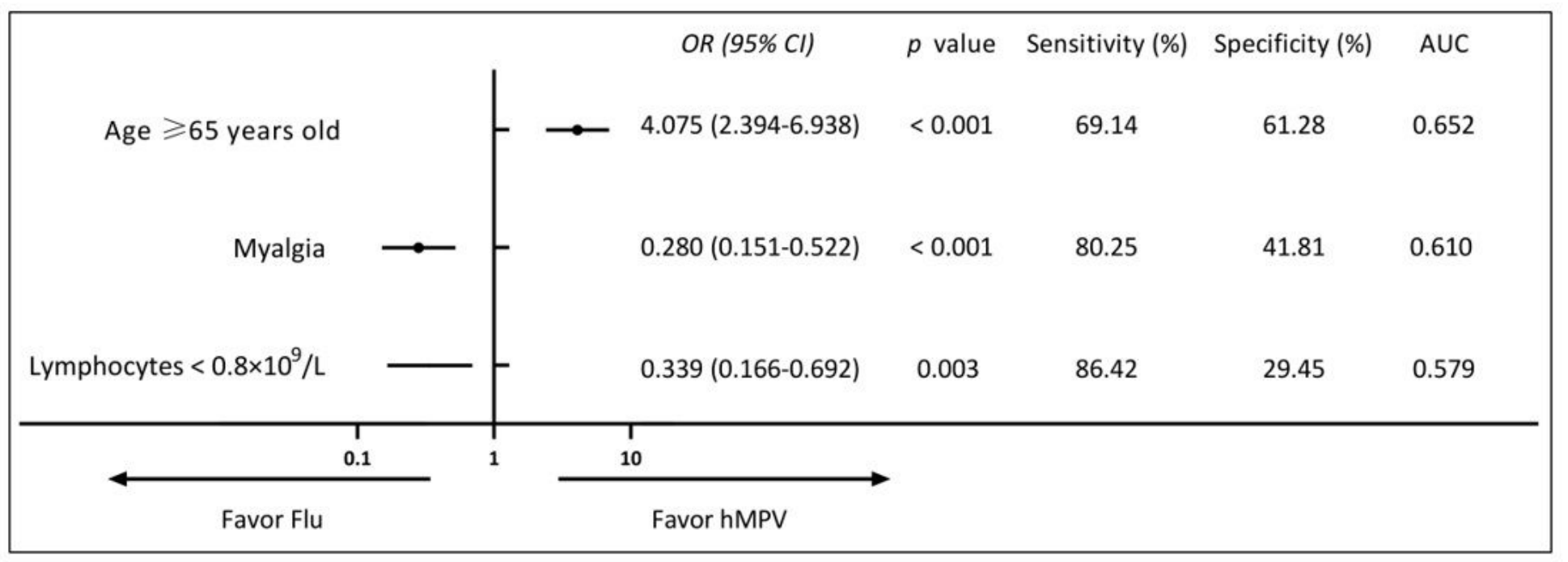

\section{Figure 3}

Predictors for Flu and hMPV infection. Age $\geq 65$ years old was positively correlated with hMPV infection, whereas myalgia and lymphocytes $<0.8 \times 109 / \mathrm{L}$ were all negatively correlated with $\mathrm{hMPV}$ infections. None of these variables exhibited good predictive performance as a means of discriminating (area under the receiver-operating characteristic curve $<0.70$ ). 


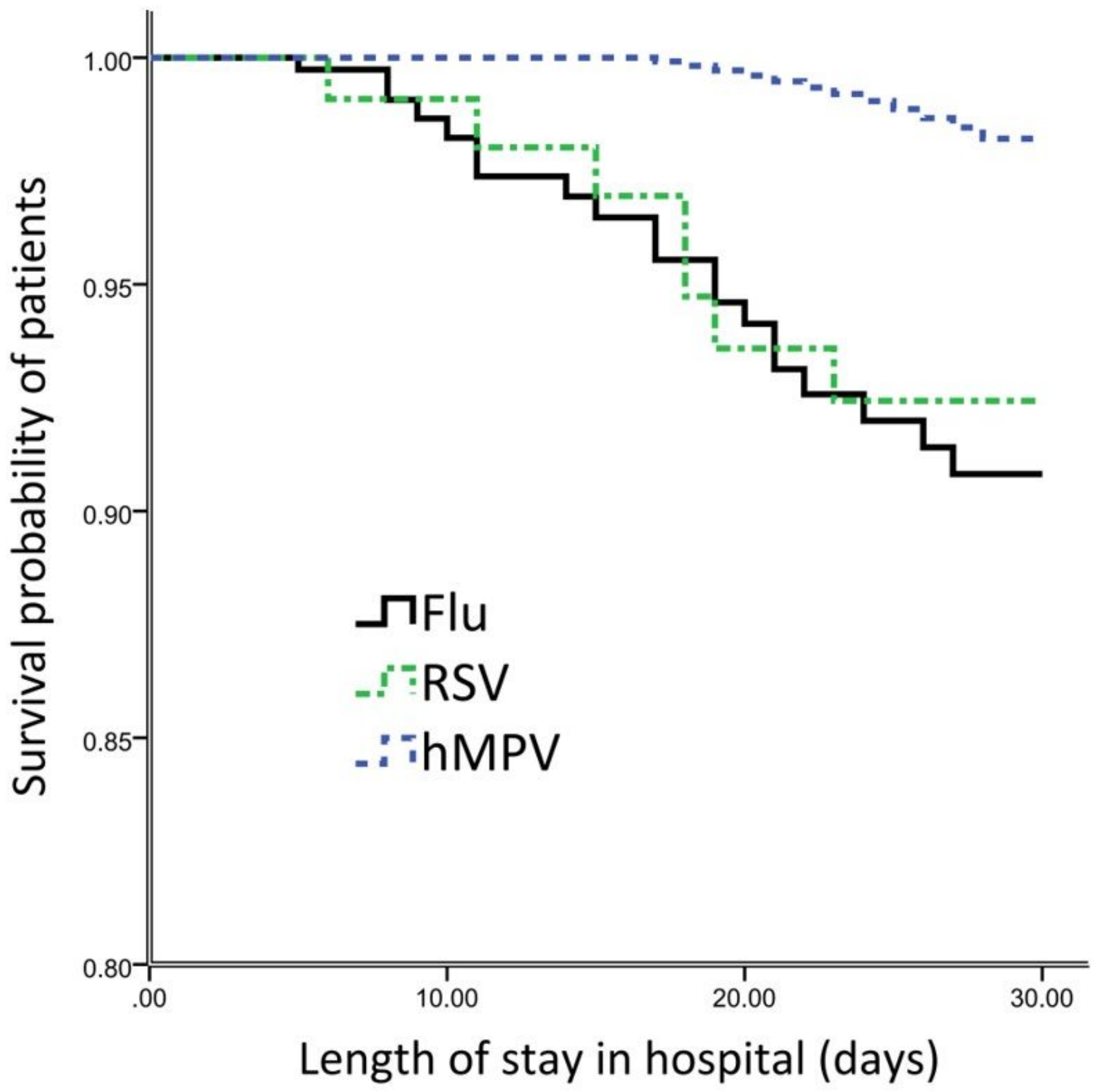

Figure 4

Survival curve of patients with pneumonia related to the three viruses (censored at $30 \mathrm{~d}$ after admission). Among patients with pneumonia, 30-day mortality rates were comparable in patients infected with RSV and influenza, and rates in both of these patient groups were significantly higher than those in patients with hMPV infections after adjusting for confounders. 


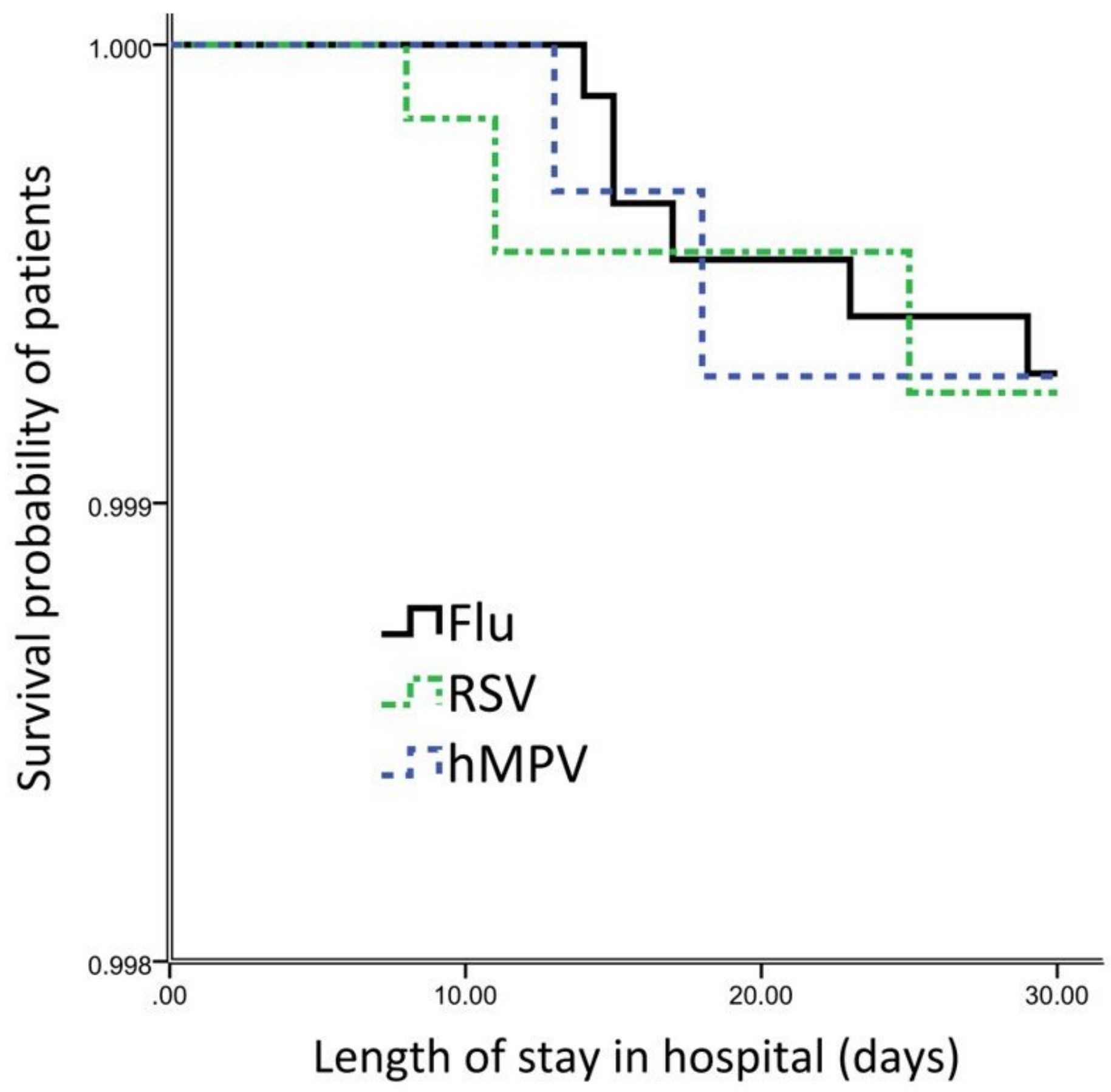

Figure 5

Survival curve of patients with the three viruses infection and without pneumonia (censored at $30 \mathrm{~d}$ after admission). Rates of 30-day mortality were similar in all patients without pneumonia infected with influenza, RSV, and hMPV infections after adjusting for confounders

\section{Supplementary Files}

This is a list of supplementary files associated with this preprint. Click to download. 
- Supplementarymaterials.docx

Page 26/26 